\title{
Plaque Formation by Vaccinia Virus in Tissue Cultures Inhibited by 5-Fluorodeoxyuridine
}

\author{
By D. BAXBY AND C. J. M. RONDLE \\ Department of Bacteriology, Liverpool University, Liverpool, England
}

(Received 5 October 1964)

\section{SUMMARY}

Vaccinia virus forms plaques in monolayers of RK 13, HEp.2 and HeLa cells grown in concentrations of 5 -fluorodeoxyuridine (FUDR) higher than those previously claimed to inhibit completely the synthesis of vaccinia virus DNA in suspended tissue cultures. The limited virus synthesis observed is not due to a varying susceptibility of the cells to FUDR, nor to the selection of virus resistant to FUDR. Experiments suggest that virus synthesis is made possible by thymidine from the DNA of dead cells.

\section{INTRODUCTION}

In many tissues 5-fluorodeoxyuridine (FUDR) blocks the conversion of deoxyuridylic acid to thymidylic acid, thus inhibiting DNA synthesis (Cohen et al. 1958). FUDR is only effective in the absence of thymidine, the latter compound annulling the inhibition (Shatkin \& Salzman, 1963). The inhibitory action of FUDR has been used in studies on the synthesis of vaccinia virus in tissue culture. It has been claimed that a concentration of $\mu \mathrm{M}$-FUDR completely inhibits vaccinia virus synthesis in suspended HeLa cells (Salzman, Shatkin \& Sebring, 1963), and HEp. 2 cells (Easterbrook, 1963). The observations made were, first, that cells infected with vaccinia virus and grown in the presence of FUDR had a virus titre after incubation for $24 \mathbf{~ h r}$ which was less than the inoculum (Salzman et al. 1963; Easterbrook, 1963); secondly, it was shown by Easterbrook that infected suspended HEp. 2 cells did not incorporate radio-active DNA precursors in the presence of FUDR. However, Gomatos et al. (1962), using monolayers of $\mathrm{L}$ cells treated with $\mu \mathrm{M}$-FUDR obtained a vaccinia virus titre which was $12 \%$ of the uninhibited control after $24 \mathrm{hr}$ incubation. Recently we have observed plaque formation by vaccinia virus in monolayers of RK 13, HEp. 2 and HeLa cells in the presence of high concentrations of FUDR. With a moderate inoculum plaque formation indicates localized virus production, and implies that virus DNA synthesis must have occurred. Such synthesis could occur if some cells in a monolayer were less susceptible to FUDR than the rest, thus supporting limited virus growth. Again, some virus particles might be more capable of replication in the presence of FUDR than others. Loddo, Schivo \& Ferrari (1963) showed that vaccinia virus resistant to the related inhibitor 5iododeoxyuridine could be developed by serial passage through human amnion cells treated with the compound. A third possibility is that localized virus synthesis, and hence plaque formation, could occur if FUDR inhibition was annulled by thymidine at discrete loci on the cell sheet. Synthesis of a DNA virus, equine 
abortion virus, in FUDR-treated $L$ cells has been recently shown to take place by the use of thymidine from the DNA of damaged cells (Gentry, Lawson \& Randall, 1964). The results given in the present paper suggest that this last possibility is the mechanism which permits limited vaccinia virus synthesis in cells inhibited by FUDR.

\section{METHODS}

Virus. The Lister Institute strain of vaccinia virus was used throughout, and until this time had been maintained solely on the rabbit dermis.

Cell lines. Three cell lines were used: the RK 13 line of transformed rabbit kidney cells (McCarthy, Taylor-Robinson \& Pillinger, 1963), the HEp.2 line of human carcinoma cells, and the HeLa line of human carcinoma cells. The last cells were provided by Dr Bruce White of Liverpool Public Health Laboratory. RK 13 and HEp. 2 cells were grown as monolayers in Parker's 199 medium containing $5 \%(v / v)$ calf serum. HeLa cells had $5 \%(v / v)$ human serum added. Incubation was at $35^{\circ}$ in an atmosphere of $5 \%(\mathrm{v} / \mathrm{v}) \mathrm{CO}_{2}$ in air.

5-fluorodeoxyuridine. FUDR (the gift of Roche Products Ltd., Welwyn Garden City, Hertfordshire) was kept as a $0 \cdot 1 \mathrm{M}$-solution at $-20^{\circ}$ and diluted to the required concentration in Eagle's tissue culture medium when needed.

Growth curves. Confluent monolayers of RK 13 cells were grown for $22 \mathrm{hr}$ in $2 \%(\mathrm{v} / \mathrm{v})$ calf serum Eagle's medium (CSE) containing $2 \mu \mathrm{M}-\mathrm{FUDR}$. Eagle's medium lacks thymidine, and this treatment depleted endogenous thymidine. The medium was removed and $10^{4}$ pock-forming units (p.f.u.) vaccinia virus allowed to absorb to each monolayer for $1 \mathrm{hr}$ at $37^{\circ}$. The monolayers were washed with CSE medium and divided into six series. One series was set aside for titration of the virus present $1 \mathrm{hr}$ after addition of the virus. CSE medium was added to oneseries and CSE medium containing 1, 3, 10 and 50 $\mu \mathrm{M}$-FUDR added to the others. Groups of monolayers from control and inhibited series were then sampled at 4, 8, 12,24 and $48 \mathrm{hr}$ after addition of virus. The medium was removed, the cells disrupted ultrasonically and titrated for vaccinia on the chorioallantois (CAM) of 12-day chick embryos by the method of McCarthy \& Dumbell (1961). The titres obtained by pock counts on the CAM were the same as those obtained by plaque counts on RK 13 monolayers.

Plaque inhibition tests. These were done on RK 13, HEp.2 and HeLa cell monolayers. Before inoculation the monolayers were treated as described above. 240 p.f.u. vaccinia virus were allowed to absorb to each monolayer for $1 \mathrm{hr}$ at $37^{\circ}$, after which time the inoculum was removed and CSE medium containing FUDR added. Duplicate sets of dishes were stained with carbol fuchsin at 24 and $48 \mathrm{hr}$, and the plaques counted. Plaque diameters were measured at $48 \mathrm{hr}$. An agar overlay was unnecessary because at the time the plaques were counted, $24 \mathrm{hr}$, secondary plaque production was negligible. The plaques in RK 13 monolayers inhibited by $50 \mu \mathrm{M}-$ FUDR were counted at $48 \mathrm{hr}$, but it is known that secondary plaque formation by vaccinia virus in these cells is not apparent until after this time (C. R. Madeley, personal communication).

Susceptibility of $R K 13$ cells to FUDR. RK 13 cells were trypsinized and distributed as monodisperse suspensions in CSE medium to 2 in. Petri dishes. Similar cells were suspended in $2 \mu \mathrm{M}$-FUDR in CSE medium. The dishes were incubated and examined daily for signs of cell division. 
Virus resistance to FUDR. High-titre vaccinia virus was passaged once through RK 13 cells grown in $10 \mu$ M-FUDR in CSE medium. The virus was harvested at $24 \mathrm{hr}$ and its plaquing ability in RK 13 cells in 10 and $20 \mu \mathrm{M}$-FUDR compared with that of the original virus stock.

The effect of dead cells on plaque formation in FUDR-treated RK 13 cells. RK 13 cells which had been grown for $22 \mathrm{hr}$ in $2 \mu \mathrm{M}$-FUDR + CSE medium were killed by one of the following methods; heating to $56^{\circ}$ for 3 min., freezing and thawing, ultrasonic disruption, and ultraviolet irradiation for $\mathbf{3 0} \mathrm{min}$. Monolayers of RK 13 cells were then infected with vaccinia in $10 \mu \mathrm{M}-\mathrm{FUDR}+\mathrm{CSE}$ medium and after $1 \mathrm{hr}$ various numbers of dead cells were added to the monolayers. The plaques were counted at $24 \mathrm{hr}$ and measured at $48 \mathrm{hr}$.

\section{RESULTS}

Growth. The growth obtained in the presence of various amounts of FUDR are shown in Table 1. This shows that with $3 \mu \mathrm{M}-F U D R$ and higher concentrations, the $24 \mathrm{hr}$ titres approached that found at $1 \mathrm{hr}$, and were significantly higher than the titre detected during eclipse. Even after incubation for $48 \mathrm{hr}$ the titres were not significantly higher than the inoculum.

Table 1. The effect of 5-fluorodeoxyuridine (FUDR) on the synthesis of vaccinia virus in RK 13 cells

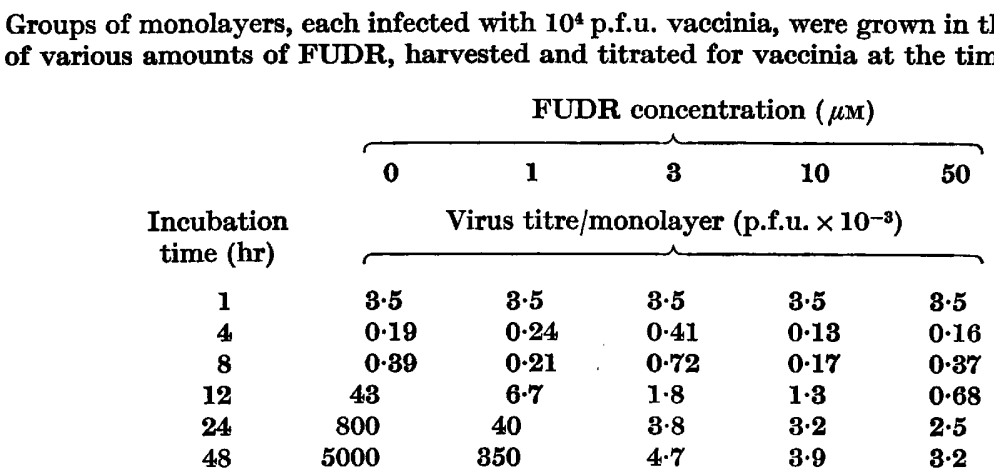

Plaque inhibition tests. In most cases plaques were seen and counted at $24 \mathrm{hr}$, but it was found more convenient to count the very small plaques produced in RK 13 cells in the presence of $50 \mu \mathrm{M}-F U D R$ at $48 \mathrm{hr}$, by which time the plaques had reached maximum size. Plaque counts are shown in Table 2. It is evident that the plaque number decreased with increasing FUDR concentrations; the plaque diameters also decreased. Uninhibited plaques in RK 13 cells were $1 \cdot 2 \mathrm{~mm}$. diameter; in $50 \mu \mathrm{M}$-FUDR they were $\mathbf{0 . 2} \mathrm{mm}$. Uninhibited plaques in the HeLa cells were $0.46 \mathrm{~mm}$. diameter; in $5 \mu \mathrm{M}$-FUDR they were $0.22 \mathrm{~mm}$. In HEp. 2 cells, vaccinia virus produced clumps of deeply staining cells and it was not possible to detect any size variation with different FUDR concentrations. Clearly in HEp. 2 and HeLa cells plaque formation occurred in FUDR concentrations higher than those previously claimed to inhibit completely vaccinia virus DNA synthesis. The results indicate that some DNA synthesis must have occurred. Therefore, the 
hypothesis suggested by Easterbrook (1963), that the recoating of inoculum virus DNA with newly synthesized protein accounts for the slight rise in titre over the eclipse level, cannot be the sole factor involved.

Susceptibility of RK 13 cells to FUDR. Monodisperse cells seeded in CSE medium settled and became attached to the glass within $24 \mathrm{hr}$, and by $48 \mathrm{hr}$ they were dividing. When $2 \mu \mathrm{M}-F U D R$ was added to the CSE medium, very few cells were attached by $48 \mathrm{hr}$, and none was seen to divide. This suggests that $2 \mu \mathrm{M}$ FUDR is sufficient to inhibit DNA replication in all RK 13 cells, and that no cells remain unaffected.

Table 2. Plaque production by vaccinia virus in monolayers of $R K 13, H E p .2$ and HeLa cells treated with 5-fluorodeoxyuridine (FUDR)

A control count of 240 plaques/monolayer was attempted. In this Table control counts have been reduced to 100 , and all other counts adjusted accordingly. Plaques were counted at $24 \mathrm{hr}$; except that plaques in RK 13 cells treated with $50 \mu \mathrm{M}-\mathrm{FUDR}$ were counted at $48 \mathrm{hr}$.

FUDR concentration $(\mu \mathrm{M})$

$\begin{array}{lccccc}\text { Cell line } & 0 & 1 & 5 & 10 & 50 \\ \text { RK 13 } & 100 & 35 & 24 & 11 & 8 \\ \text { HEp.2 } & 100 & 49 & 25 & 8 & \text { N.T.* } \\ \text { HeLa } & 100 & 17 & 4 & \dagger & \text { N.T. }\end{array}$

* N.T. = not tested; $†$ Unequivocal demonstration of plaques not possible.

Virus resistance to FUDR. There was no increased resistance to FUDR in vaccinia which had been passaged once in the presence of $10 \mu \mathrm{M}$-FUDR. Plaque production by such virus was decreased by $91 \%$ in $10 \mu \mathrm{M}$ and by $96 \%$ in $20 \mu \mathrm{M}$-FUDR. That of the original virus was decreased by $88 \%$ in $10 \mu \mathrm{M}$ and $91 \%$ in $20 \mu \mathrm{M}$-FUDR. Hence it is unlikely that vaccinia virus synthesis in FUDR-treated cells was initiated by virus resistant to FUDR.

The effect of dead cells on plaque formation in FUDR-treated RK 13 cells. Trypan Blue staining showed that about $1.2 \%$ of the RK 13 cells were dead after $22 \mathrm{hr}$ in $2 \mu \mathrm{M}$-FUDR + CSE medium. Such monolayers infected with 160 p.f.u. vaccinia in $10 \mu \mathrm{M}$-FUDR developed 14 plaques of $0.4 \mathrm{~mm}$. maximum diameter. Thymidinedepleted dead cells were added to similar monolayers and when the number of dead cells added was the same as the number of dead cells originally present, there were 25 plaques of maximum diameter $0.45 \mathrm{~mm}$. When ten times the original number of dead cells were added there were 53 plaques of maximum diameter $0.56 \mathrm{~mm}$. These results suggest that thymidine from the DNA of dead cells was used for vaccinia virus synthesis, and that there were sufficient dead cells in a monolayer to support the limited virus synthesis observed in high concentrations of FUDR. Shatkin \& Salzman (1963) suggested that any thymidine available will be used preferentially for virus synthesis.

\section{DISCUSSION}

Examination of Tables 1 and 2 shows an apparent discrepancy between the results obtained by the two methods used, namely, total virus counts and plaque counts. In $10 \mu \mathrm{M}-\mathrm{FUDR}$, for example, only $0.1 \%$ virus synthesis was indicated by 
the growth curve, whereas the number of plaques produced was $11 \%$ as compared to the control. However, the latter estimate is of plaque number only and does not take into account the smaller area of necrosis, and the smaller virus yield/cell. The area of a plaque in $10 \mu \mathrm{M}-F U D R$ was $0.12 \mathrm{sq} . \mathrm{mm}$. as compared to $1.1 \mathrm{sq} . \mathrm{mm}$. for the control; hence the discrepancy was decreased from a 100-fold to 10-fold. This suggests that $10 \mu \mathrm{M}$-FUDR decreased the virus yield/cell to about $10 \%$ of that of the control, but that in spite of the lower yield of virus, cell necrosis was found in inhibited cultures. A similar relationship was shown for other concentrations of FUDR. The results presented indicate that complete inhibition of vaccinia virus synthesis by FUDR is difficult, if not impossible, to obtain in monolayer culture. Dead cells in such cultures may act as sources of thymidine which may permit the formation of plaques. It is possible that complete inhibition may be easier to achieve in suspended cultures since any thymidine present will be rapidly dispersed and diluted. It appears that virus synthesis in the presence of FUDR may not be limited to vaccinia virus. The synthesis of bovine abortion virus by the mechanism suggested above has already been mentioned (Gentry et al. 1964). Lam \& Atherton (1963) only found $93 \%$ plaque inhibition of herpes simplex virus in $\mathrm{L}$ cells inhibited by $80 \mu \mathrm{M}$-FUDR. Undoubtedly FUDR markedly decreases the titre of vaccinia virus in tissue culture. In view of the results presented here, however, care must be taken when interpreting experiments on viral protein synthesis in FUDR-treated tissue cultures (Salzman et al. 1963), since complete inhibition of infective virus may not have been achieved.

We wish to thank Professor K. R. Dumbell and Professor A. W. Downie, F.R.S. for helpful discussions during this work.

\section{REFERENCES}

Cohen, S. S., Flaks, J. S., Barner, H. D., Loeb, M. R. \& Lichtenstein, J. (1958). The mode of action of 5-fluorouracil and its derivatives. Proc. natn. Acad. Sci., Wash. 44, 1004.

EASTERBROoK, K. B. (1963). Conservation of vaccinial DNA during an abortive cycle of multiplication. Virology, 21, 508.

Gentry, G. A., Lawson, L. \& Randall, C. C. (1964). Replication of a DNA virus in the presence of an inhibitor of DNA synthesis. Fed. Proc. 23, 131.

Gomatos, P. J., Tamm, I., Dales, S. \& Franklin, R. M. (1962). Reovirus type 3: physical characteristics and interaction with $L$ cells. Virology, 17, 441.

LAM, K. S. K. \& Atherton, J. G. (1963). Measles virus. Nature, Lond. 197, 820.

LodDo, B., Schivo, M. L. \& Ferrari, W. (1963). Development of vaccinia virus resistant to 5-iodo-2-deoxyuridine. Lancet, ii, 914.

MCCARTHY, K. \& Dumbell, K. R. (1961). Chorioallantoic inoculation of eggs-improved method. Virology, 14, 488.

McCarthy, K., Taylor-Robinson, C. H. \& Pillinger, S. (1963). Isolation of rubella virus from cases in Great Britain. Lancet, ii, 593.

Satzman, N. P., Shatkin, A. J. \& Sebring, E. D. (1963). Viral protein and DNA synthesis in vaccinia virus-infected HeLa cell cultures. Virology, 19, 542.

Shatkin, A. J. \& Salzman, N. P. (1963). Deoxyribonucleic acid synthesis in vaccinia virus-infected HeLa cells. Virology, 19, 551. 
